CMIP6 SWE ensemble mean

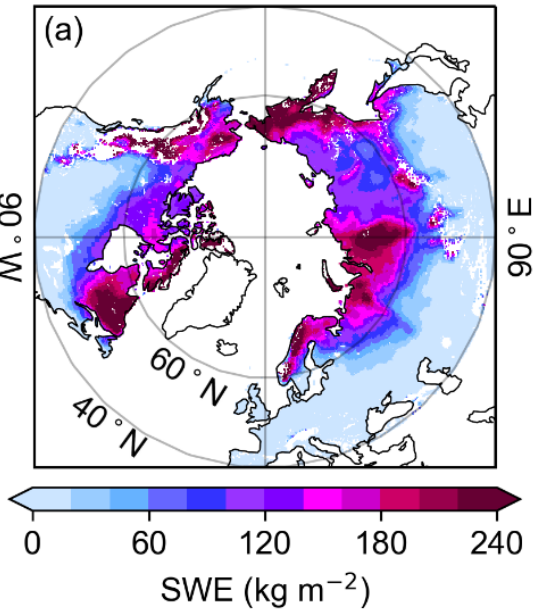

CMIP6 P ensemble mean

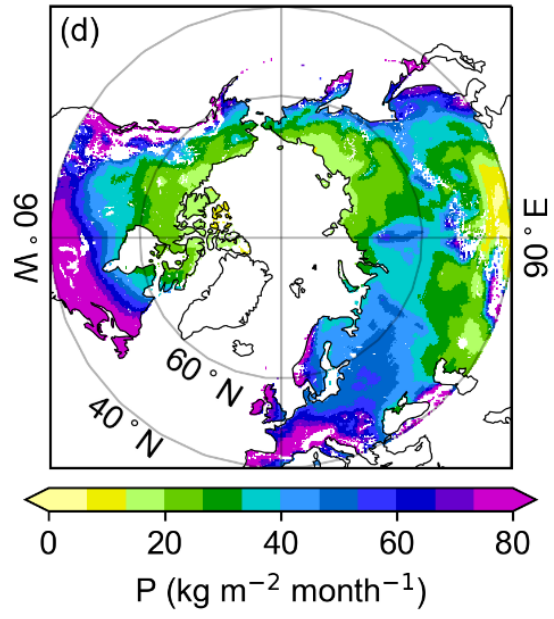

CMIP6 T ensemble mean

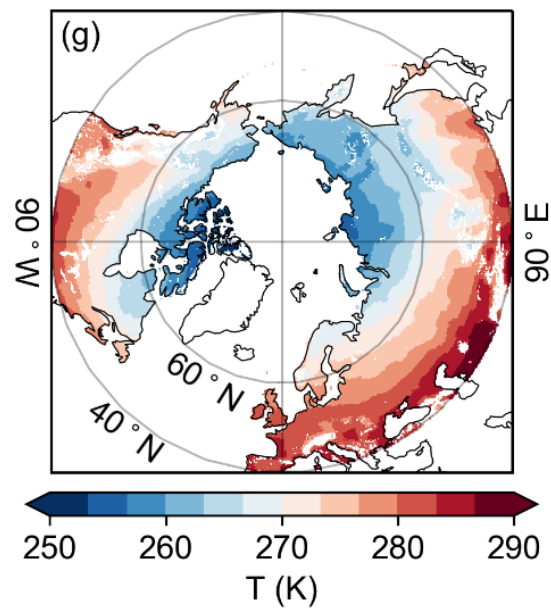

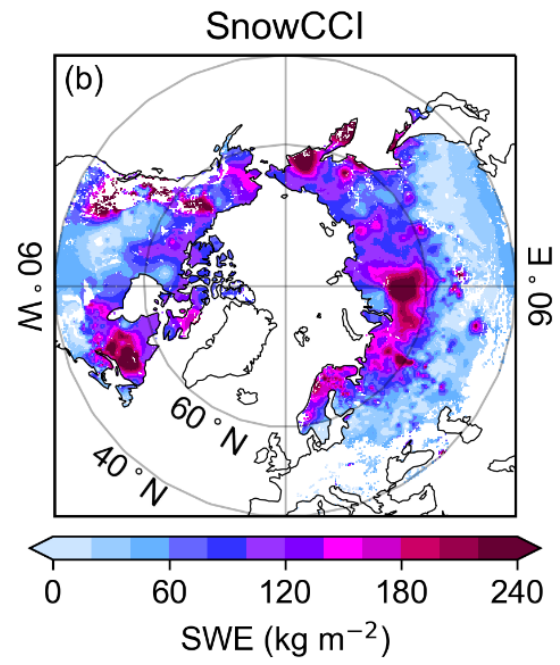
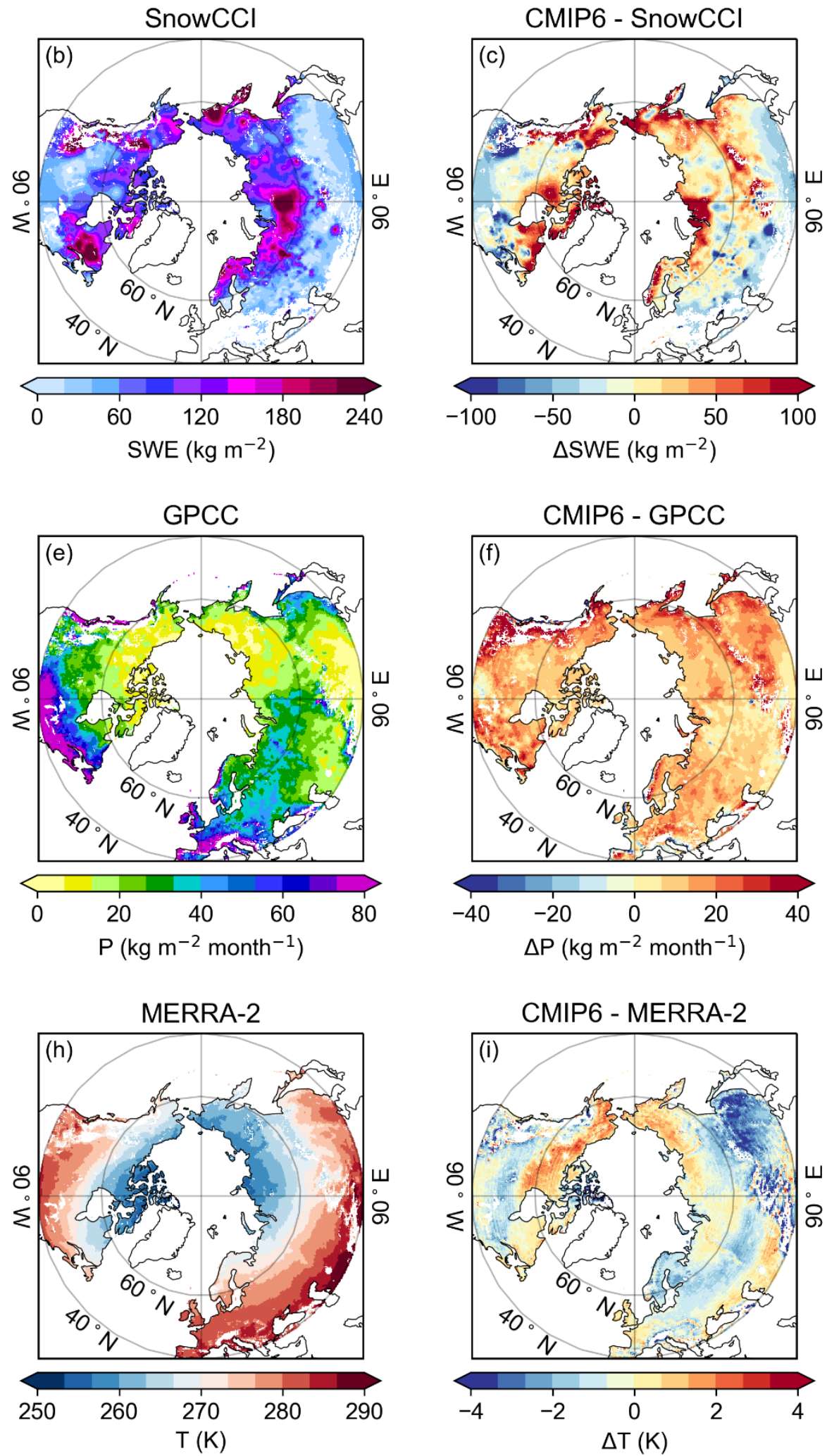

Figure S1 (top) Mean SWE in April for CMIP6 multi-model ensemble mean, SnowCCI and the difference CMIP6SnowCCI for the period 1982-2014. (middle) Mean P in April for CMIP6 multi-model ensemble mean, GPCC and the difference CMIP6-GPCC for the period 1982-2014. (bottom) Mean T in April for CMIP6 multi-model ensemble mean, MERRA-2 and the difference CMIP6-MERRA-2 for the period 1982-2014. 


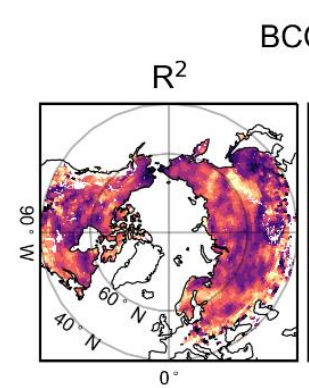

BCC-CSM2-MR historical

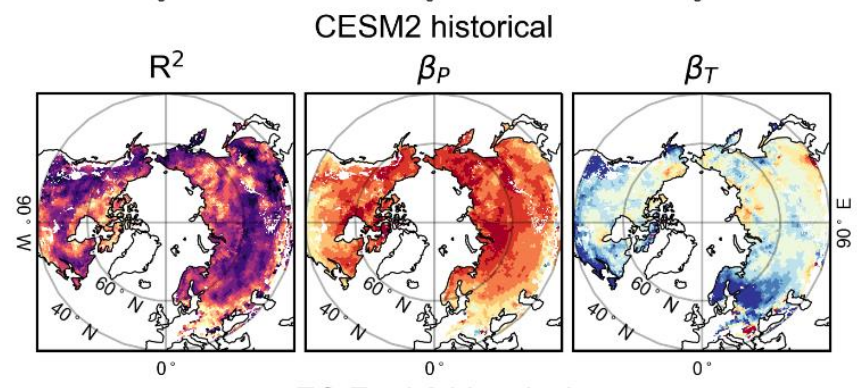

EC-Earth3 historical

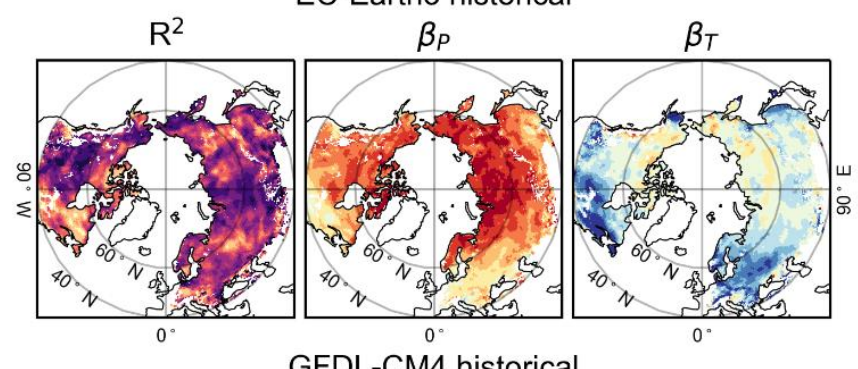

GFDL-CM4 historical

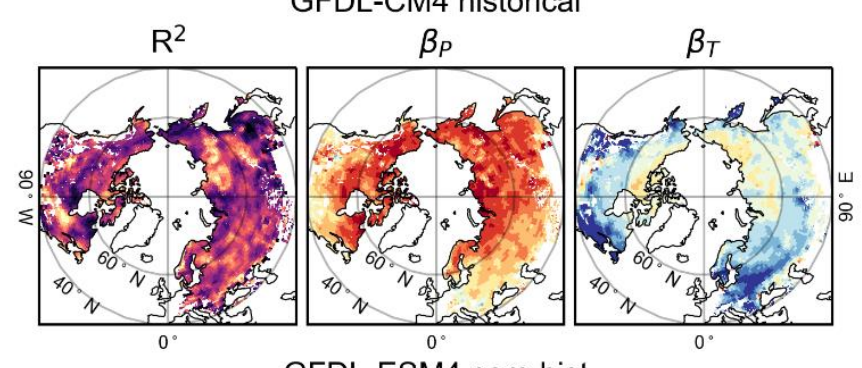

GFDL-ESM4 esm-hist

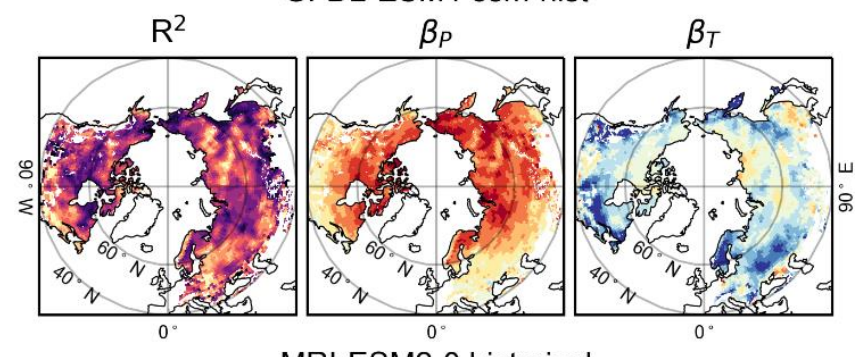

MRI-ESM2-0 historical

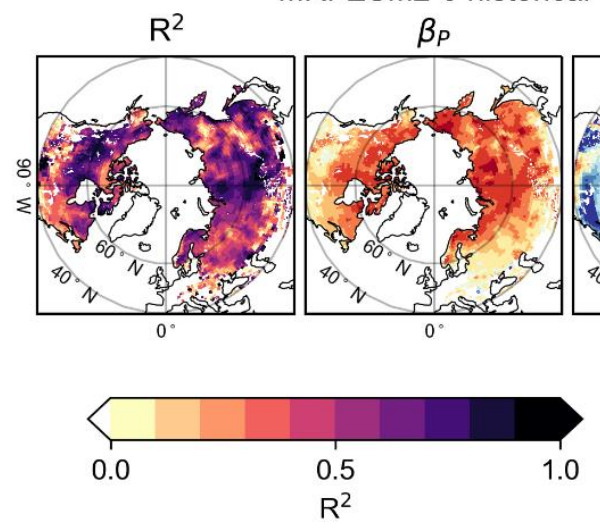

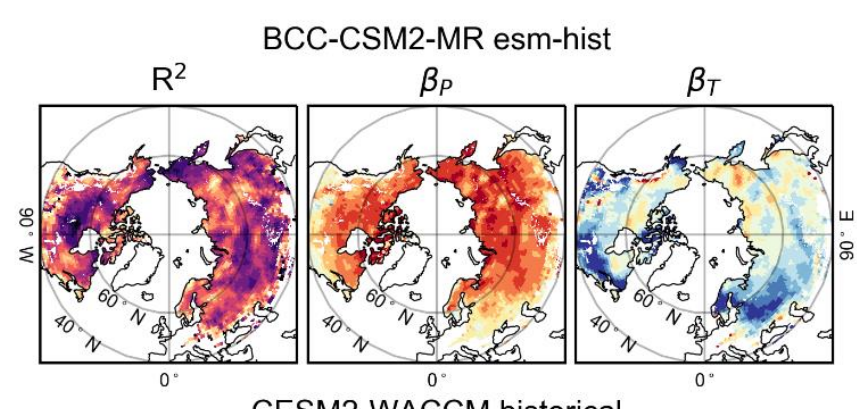

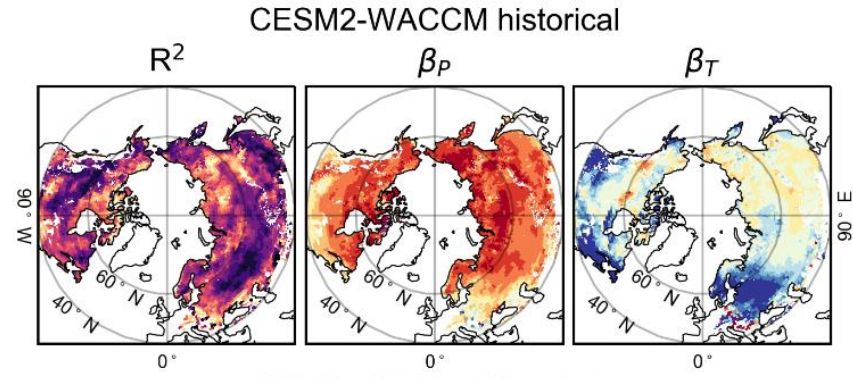

EC-Earth3-Veg historical

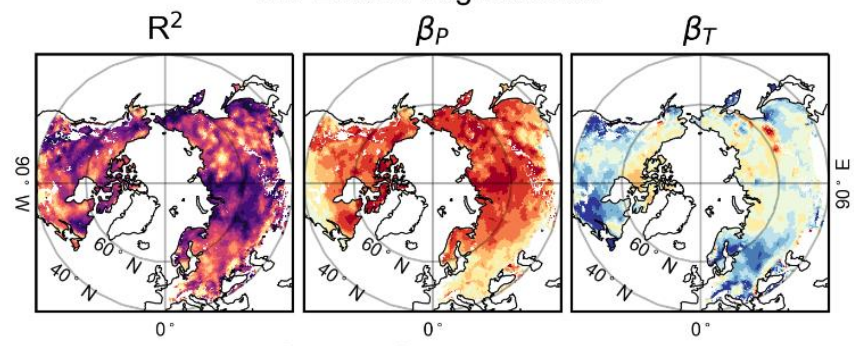

GFDL-ESM4 historical

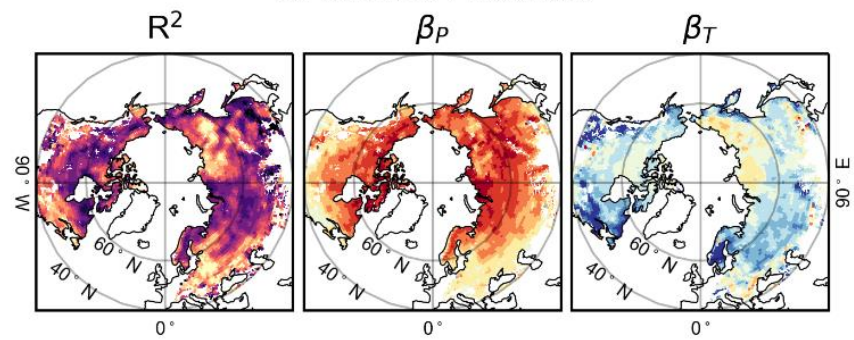

MPI-ESM1-2-HR historical

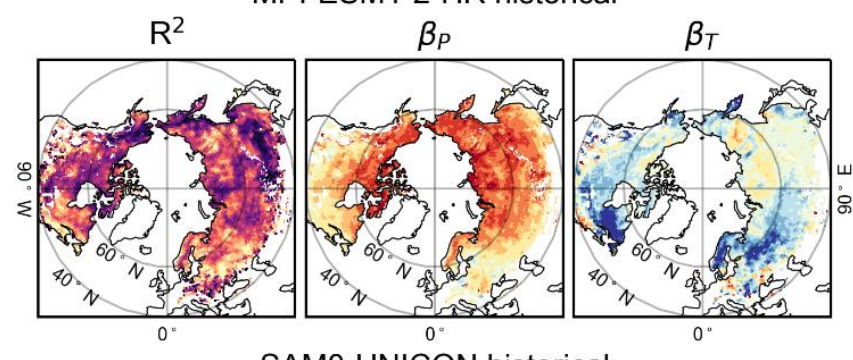

SAMO-UNICON historical
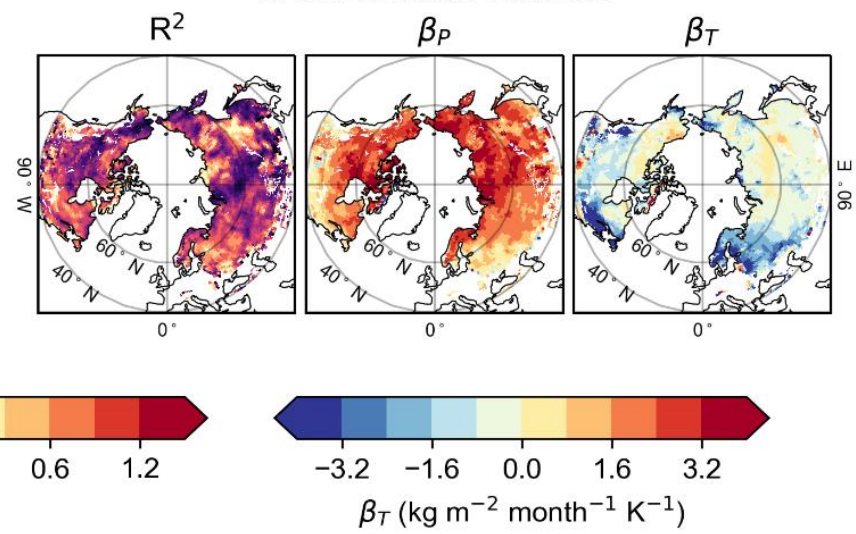

Figure S2. Linear regression parameters $\mathrm{R}^{2}, \beta_{\mathrm{P}}$, and $\beta_{\mathrm{T}}$ in winter 1982-2014. 
BCC-CSM2-MR historical

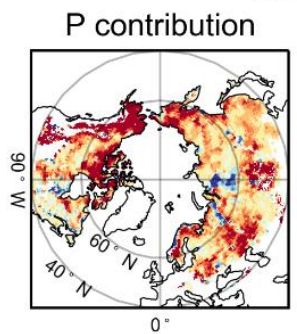

T contribution
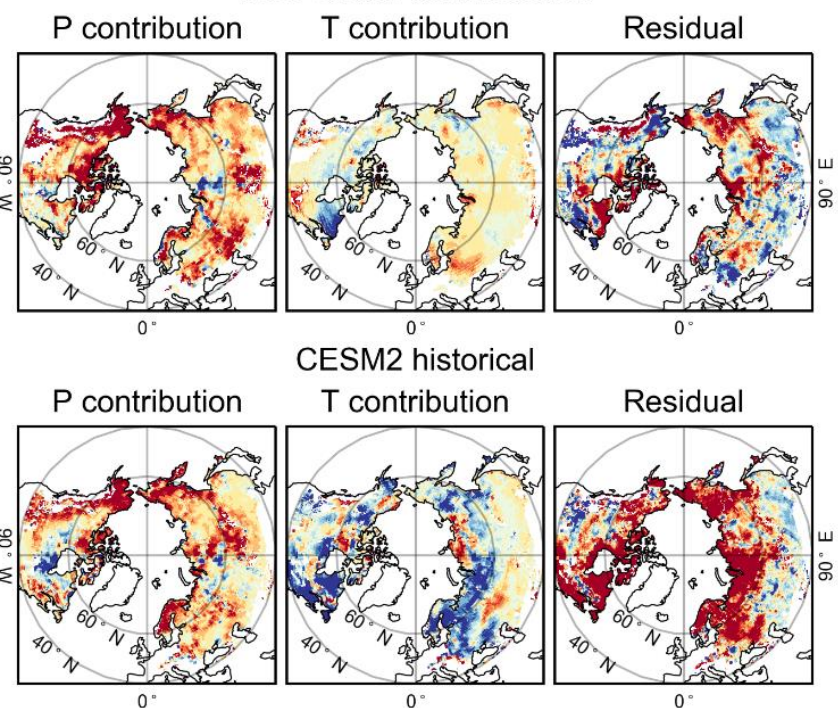

T contribution

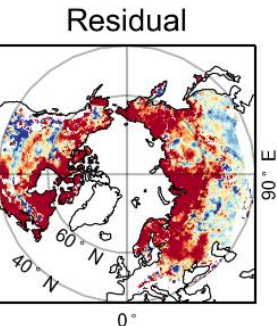

EC-Earth3 historical

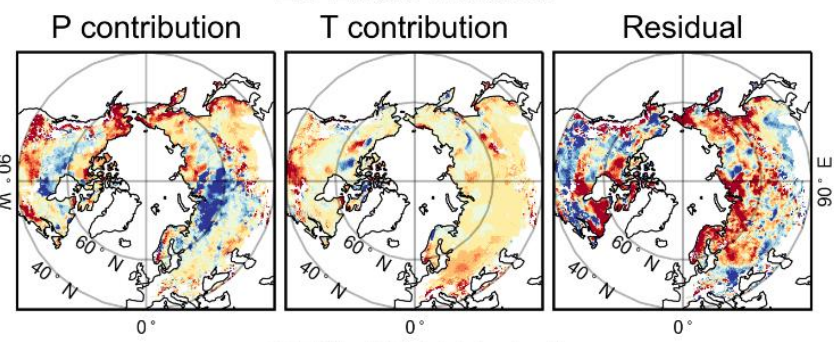

GFDL-CM4 historical

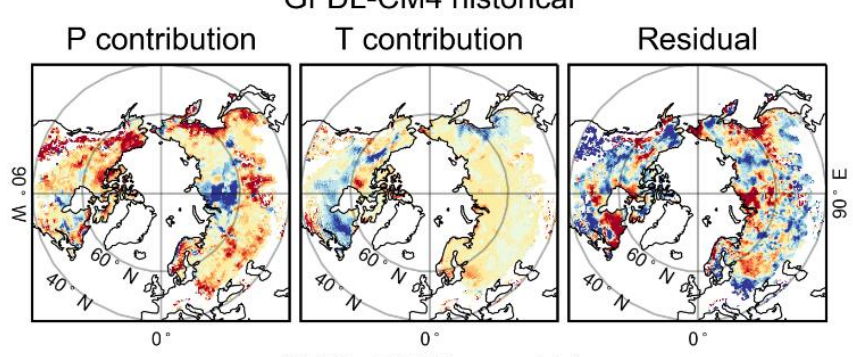

GFDL-ESM4 esm-hist

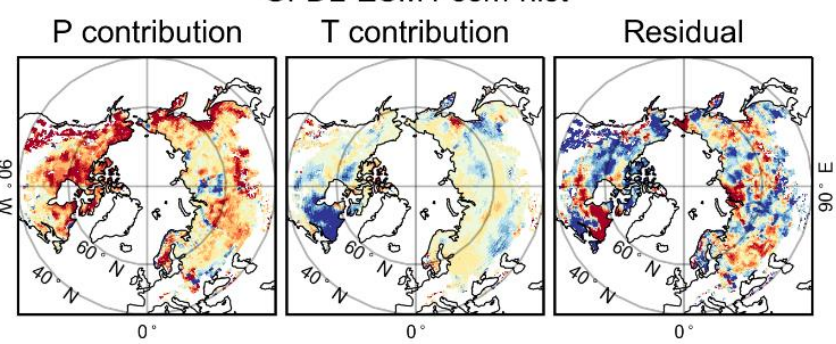

MRI-ESM2-0 historical
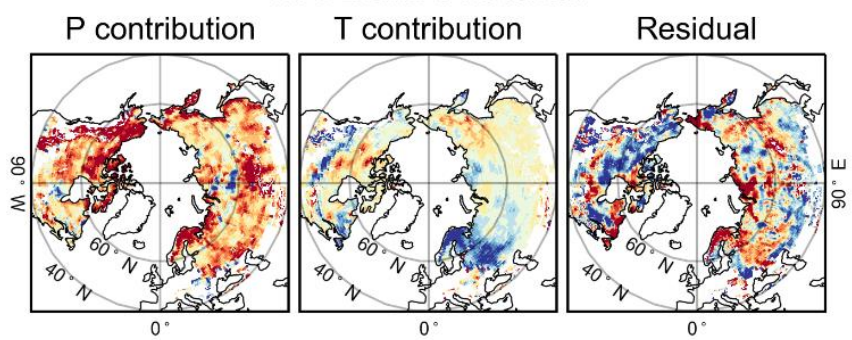

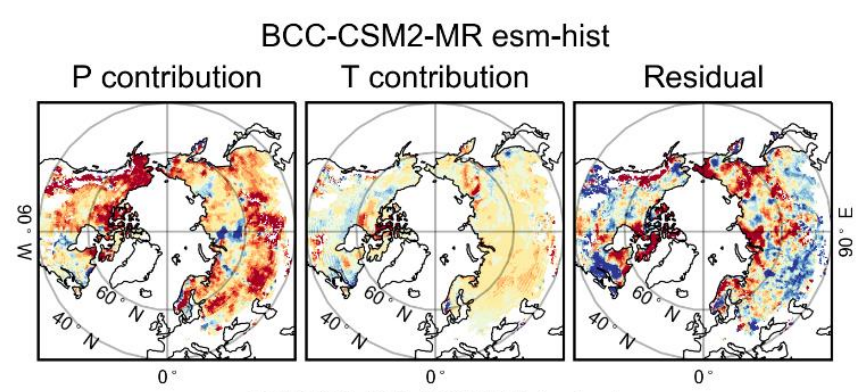

CESM2-WACCM historical

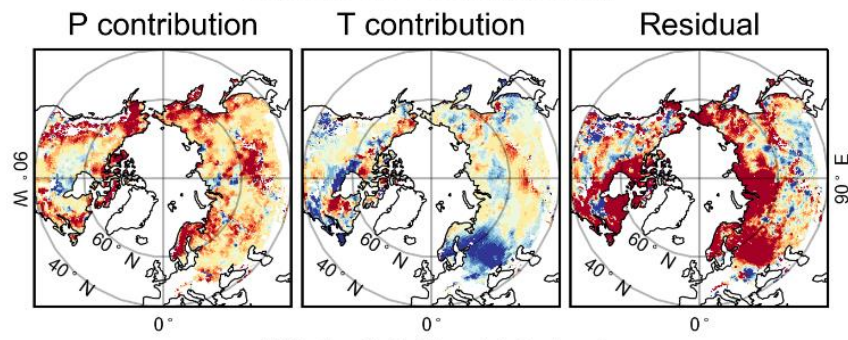

EC-Earth3-Veg historical

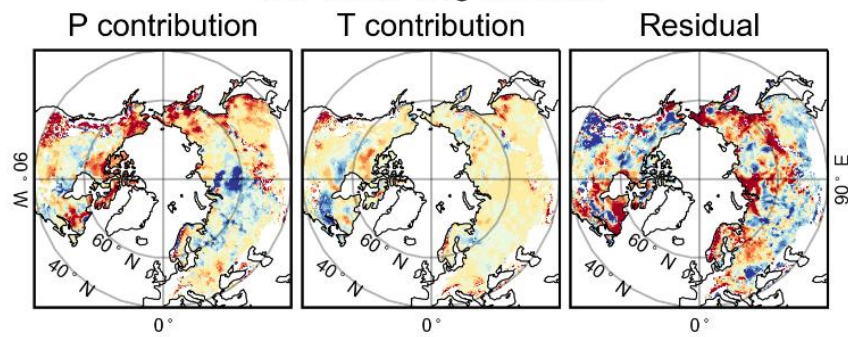

GFDL-ESM4 historical

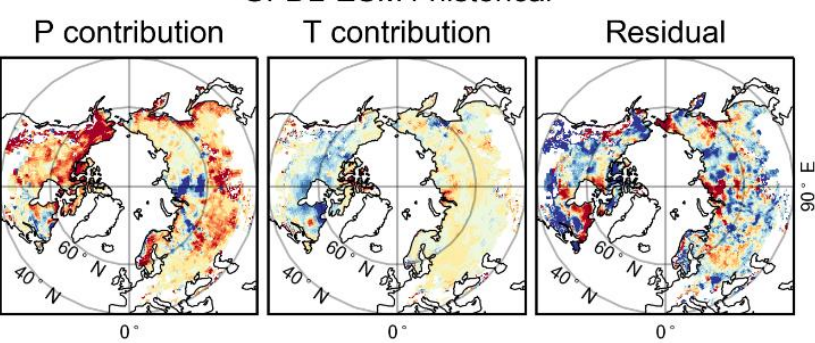

MPI-ESM1-2-HR historical
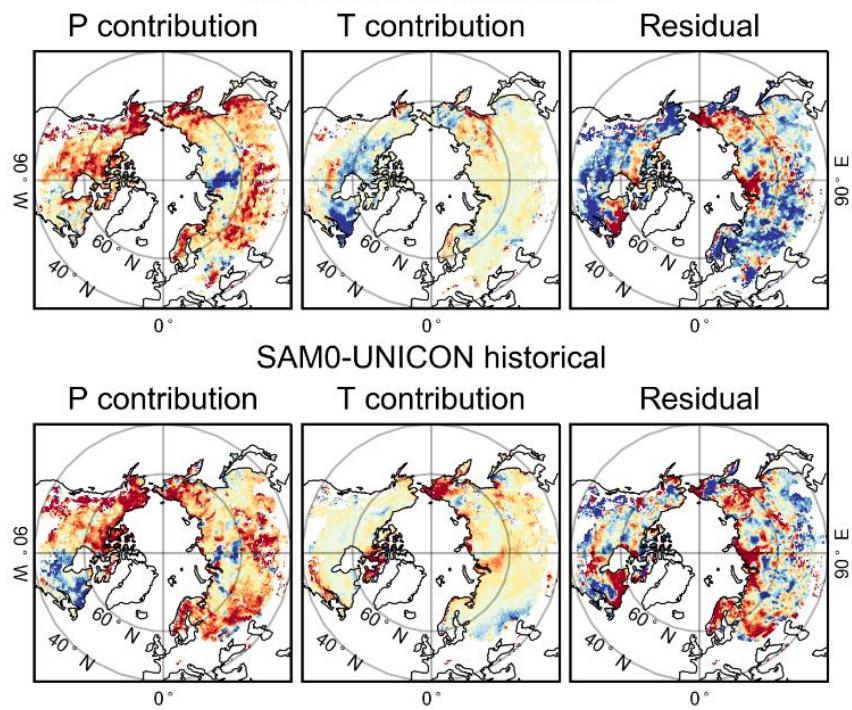

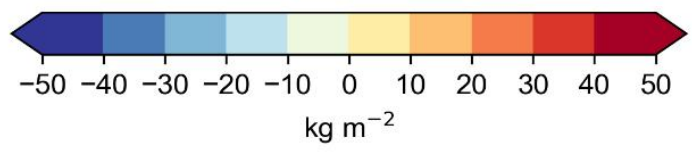

Figure S3. Spatial distribution of $\mathrm{P}$ contribution, T contribution and constant for each model in winter 1982-1991. 
BCC-CSM2-MR historical

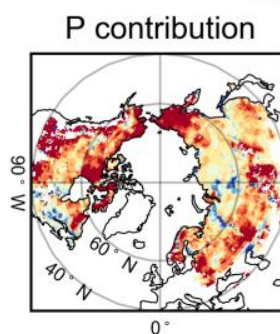

$T$ contribution
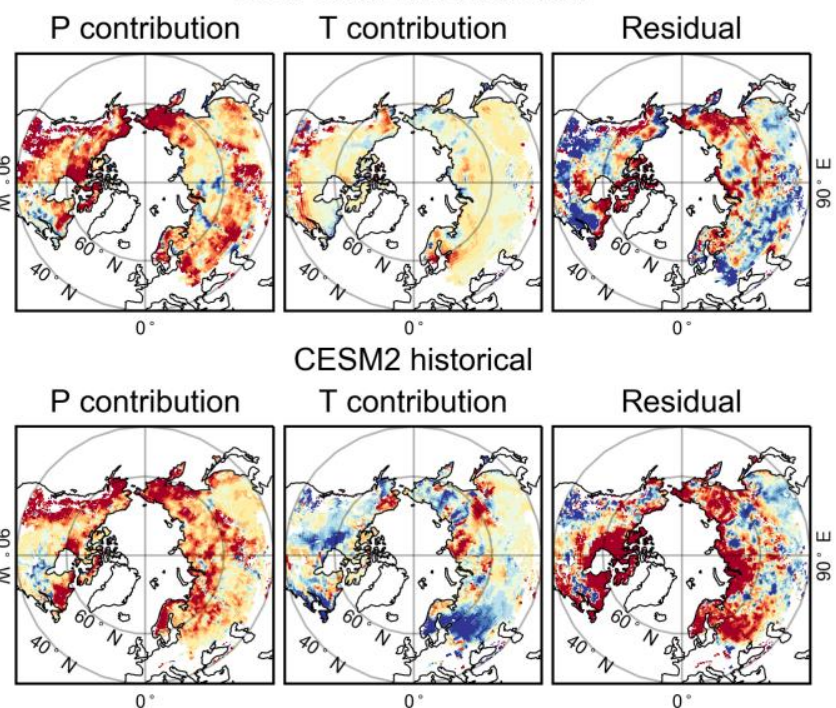

T contribution

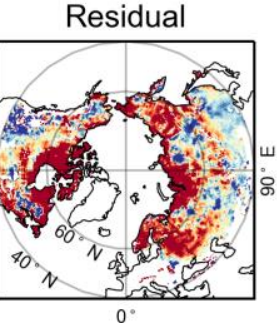

\section{EC-Earth3 historical}
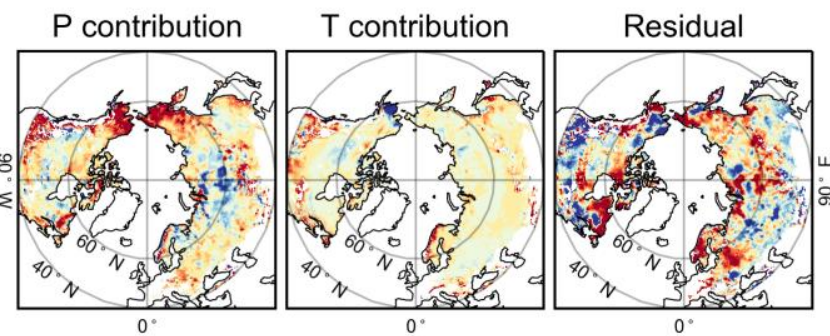

GFDL-CM4 historical

P contribution $\mathrm{T}$ contribution
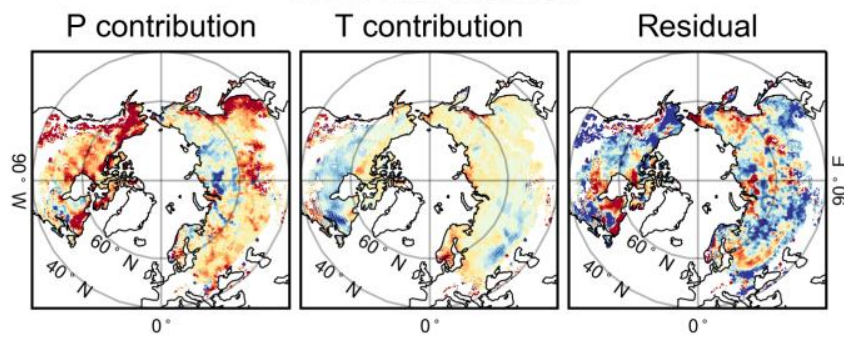

GFDL-ESM4 esm-hist

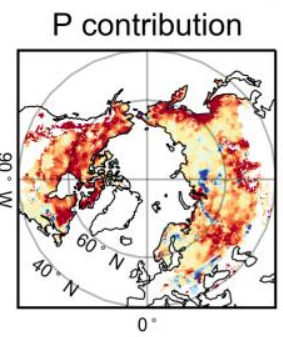

$T$ contribution

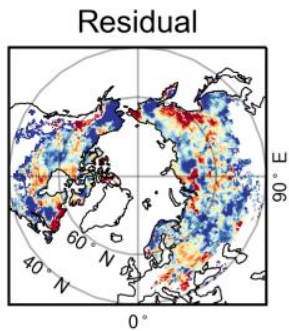

MRI-ESM2-0 historical
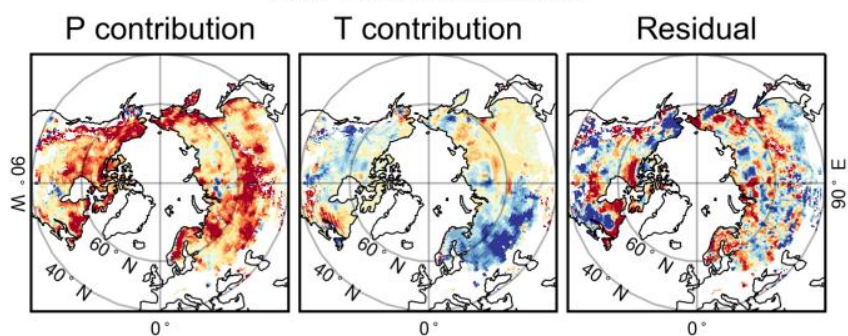

BCC-CSM2-MR esm-hist

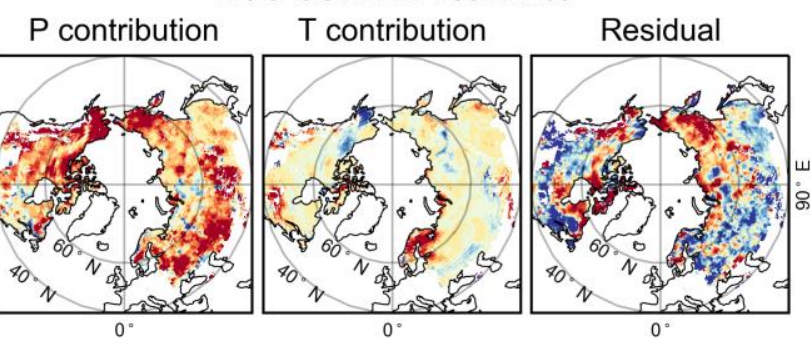

CESM2-WACCM historical

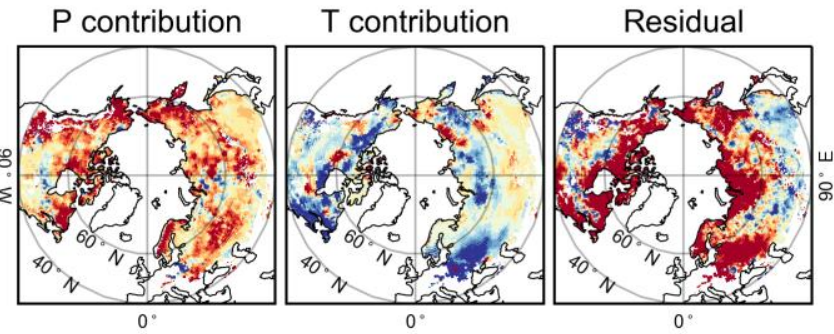

EC-Earth3-Veg historical

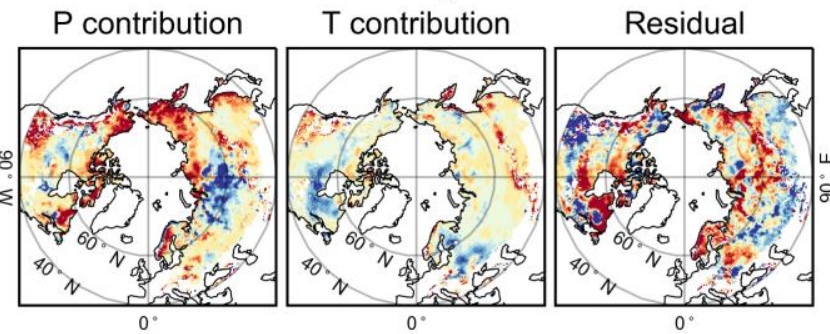

GFDL-ESM4 historical

P contribution T contribution Residual

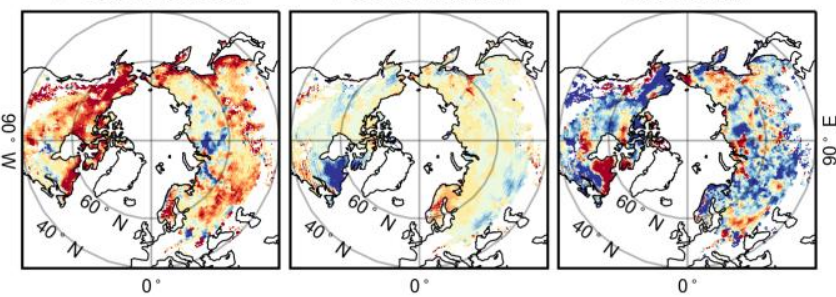

MPI-ESM1-2-HR historical
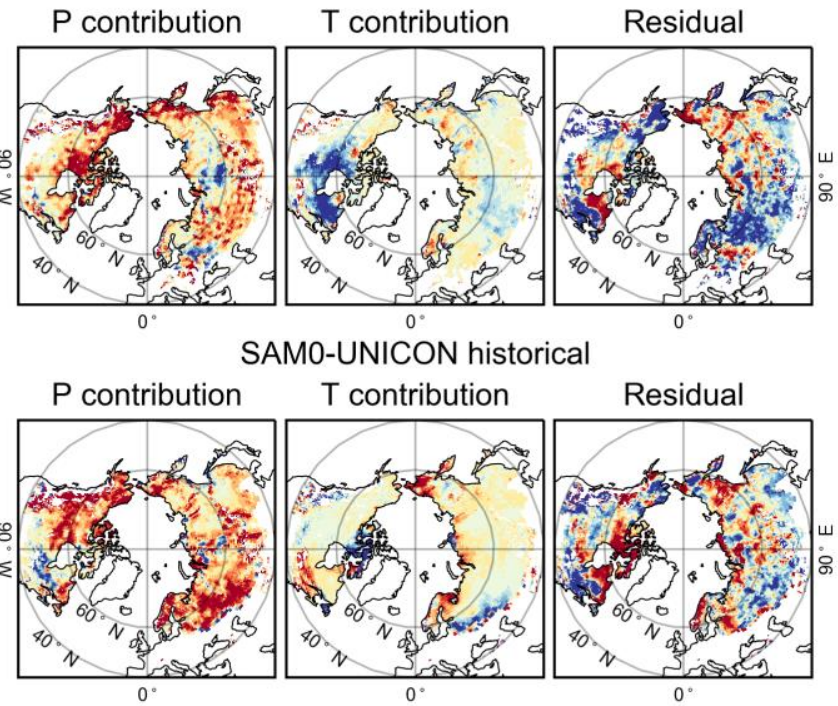

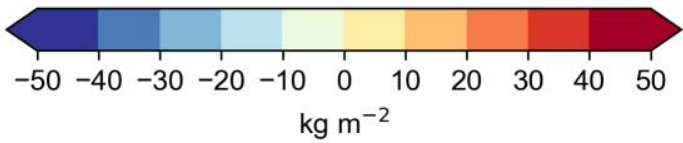

Figure S4. Spatial distribution of P contribution, T contribution and constant for each model in winter 1992-2001. 
BCC-CSM2-MR historical

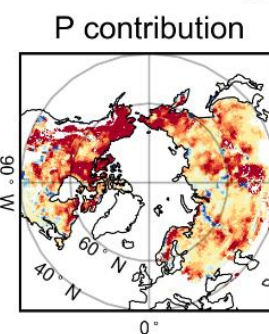

$T$ contribution
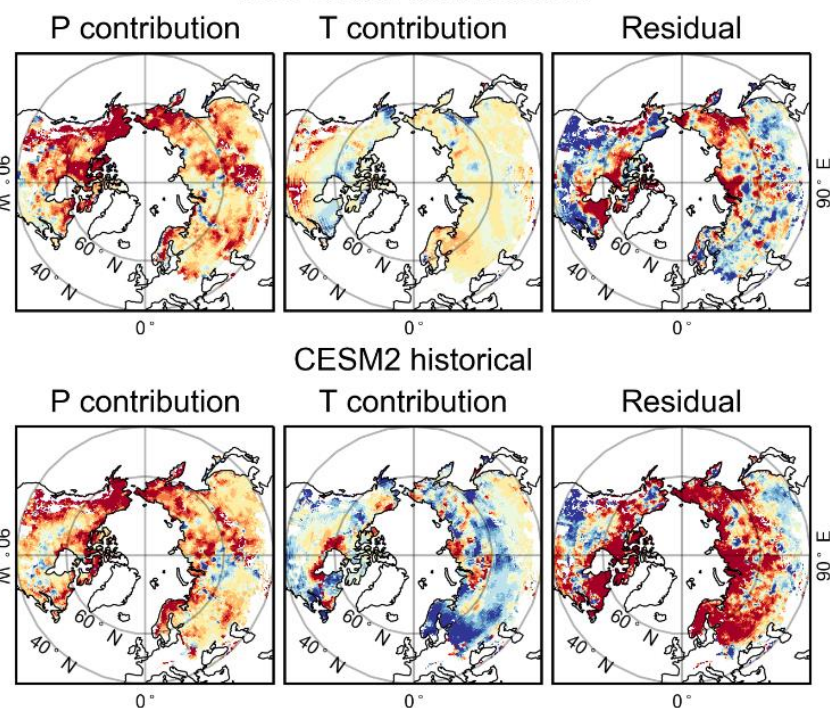

T contribution

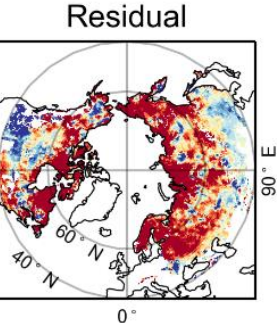

\section{EC-Earth3 historical}
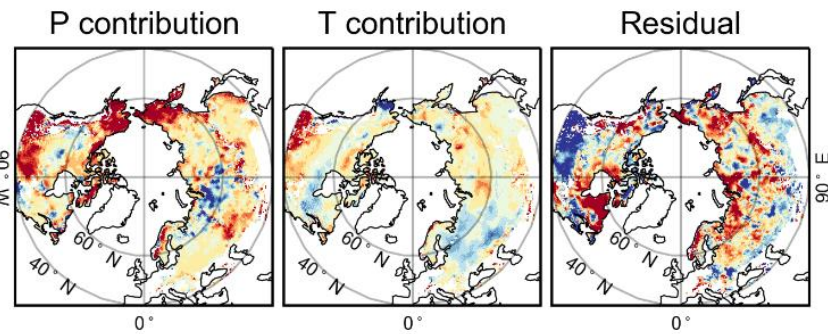

GFDL-CM4 historical

$\mathrm{P}$ contribution $\mathrm{T}$ contribution
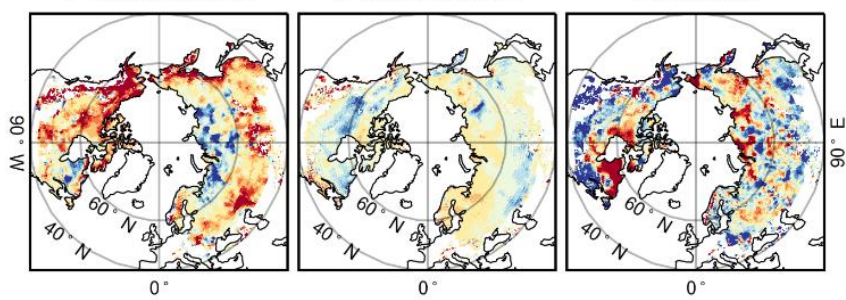

GFDL-ESM4 esm-hist

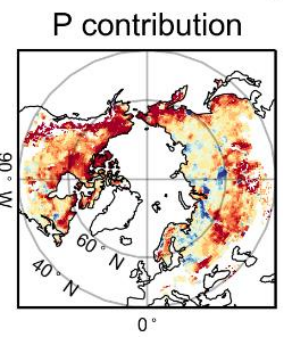

$T$ contribution

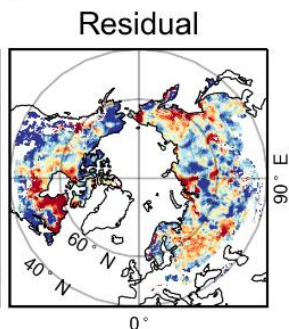

MRI-ESM2-0 historical
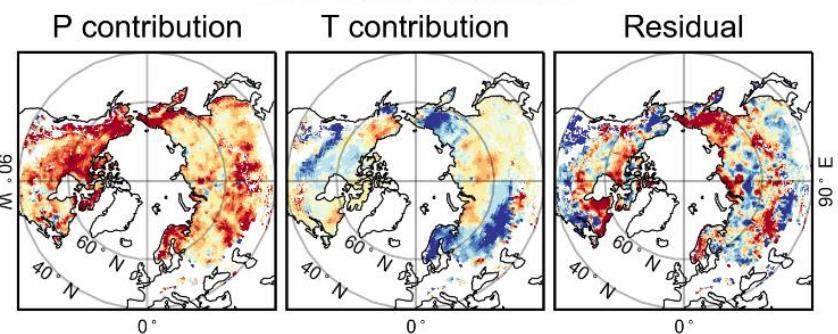

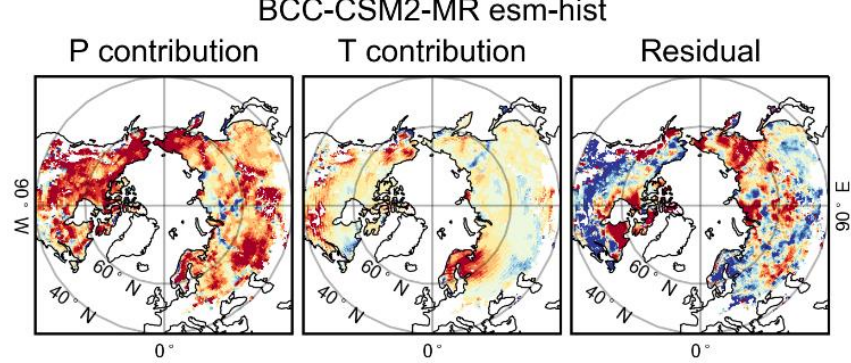

CESM2-WACCM historical

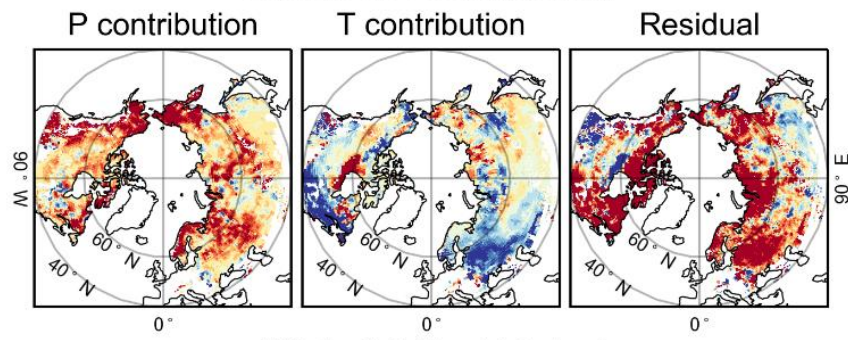

EC-Earth3-Veg historical

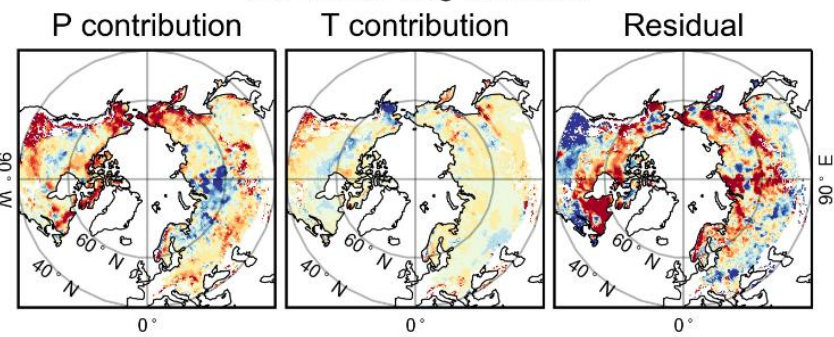

GFDL-ESM4 historical

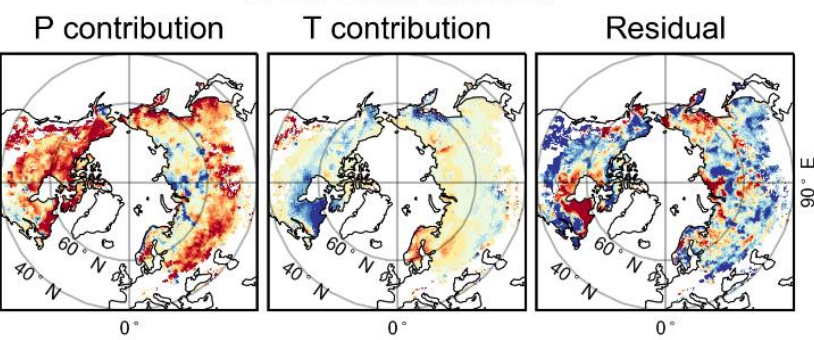

MPI-ESM1-2-HR historical
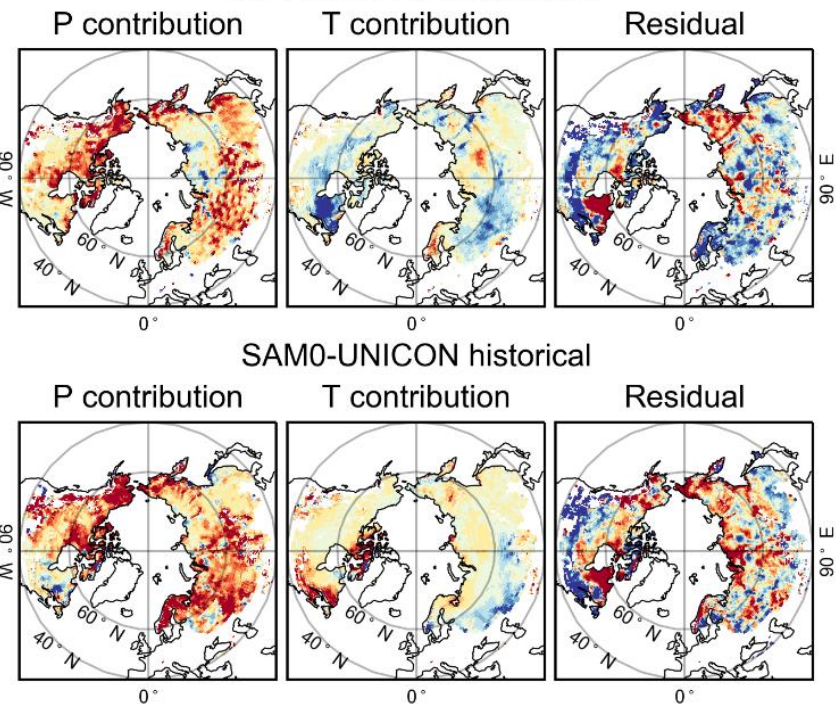

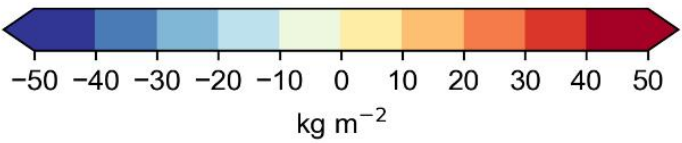

Figure S5. Spatial distribution of P contribution, T contribution and constant for each model in winter 2002-2014. 
BCC-CSM2-MR historical
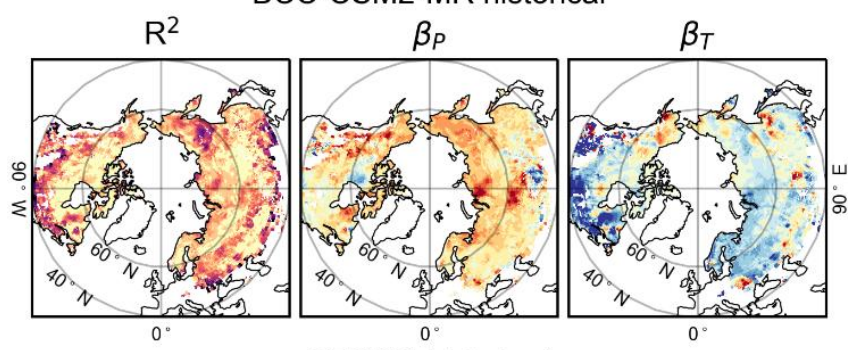

CESM2 historical
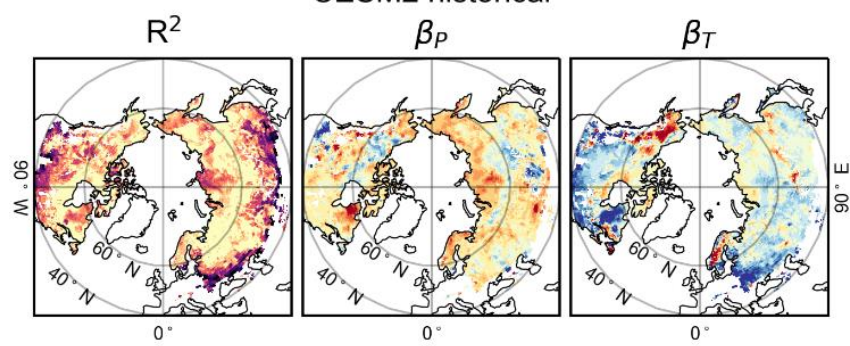

EC-Earth3 historical

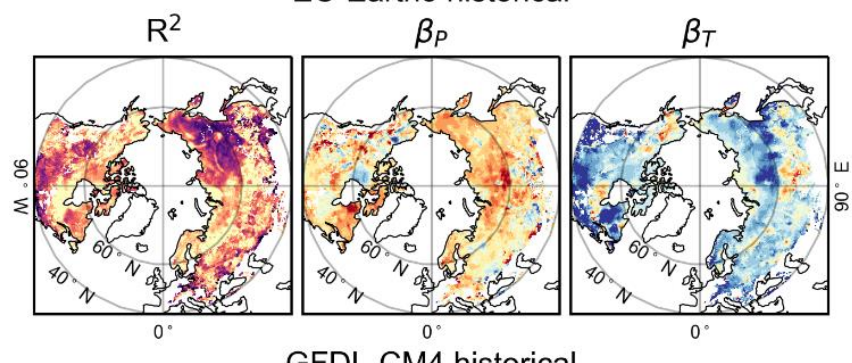

GFDL-CM4 historical

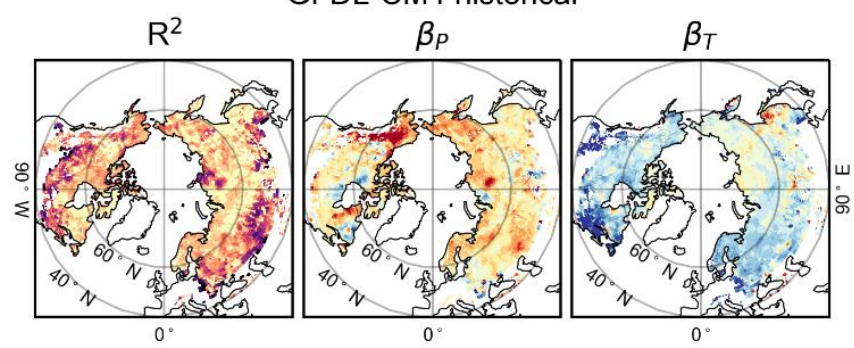

GFDL-ESM4 esm-hist

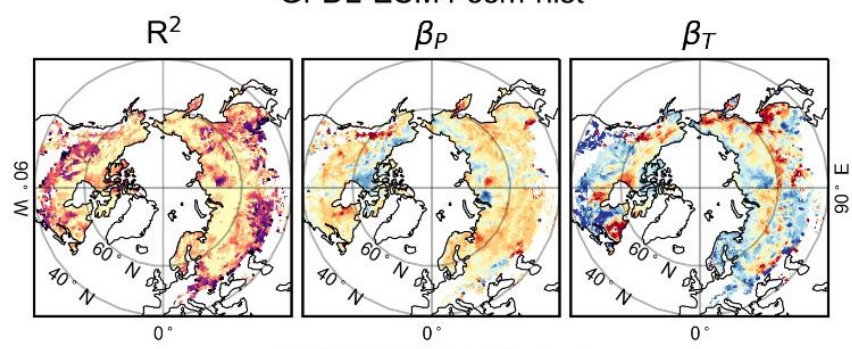

MRI-ESM2-0 historical
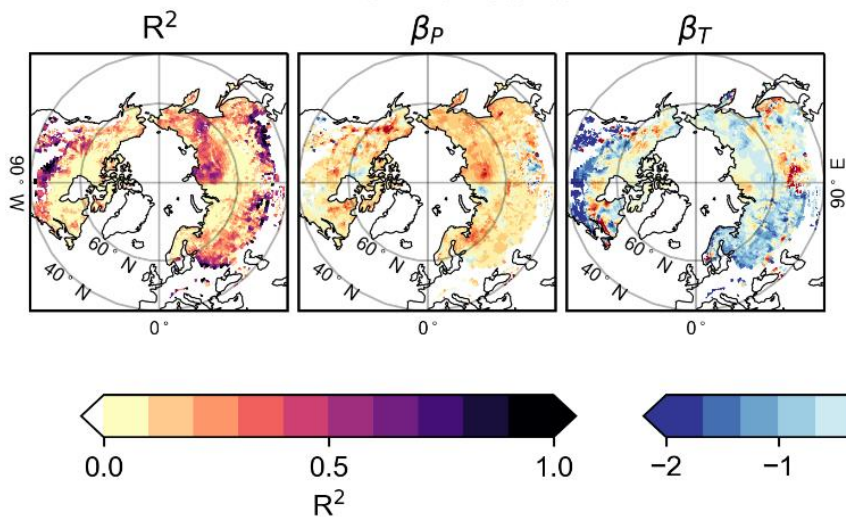

BCC-CSM2-MR esm-hist

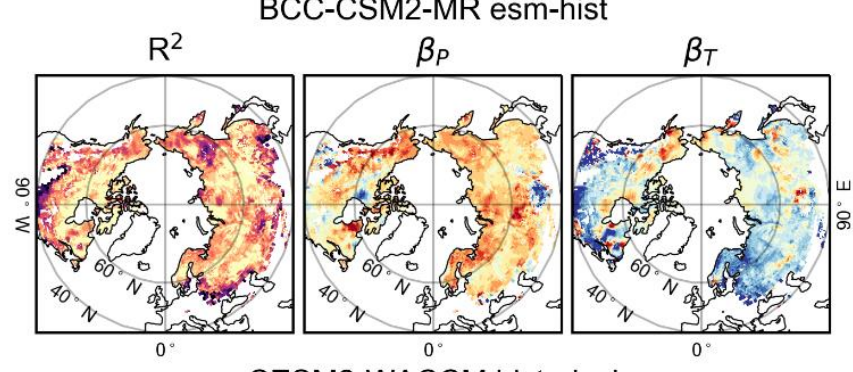

CESM2-WACCM historical

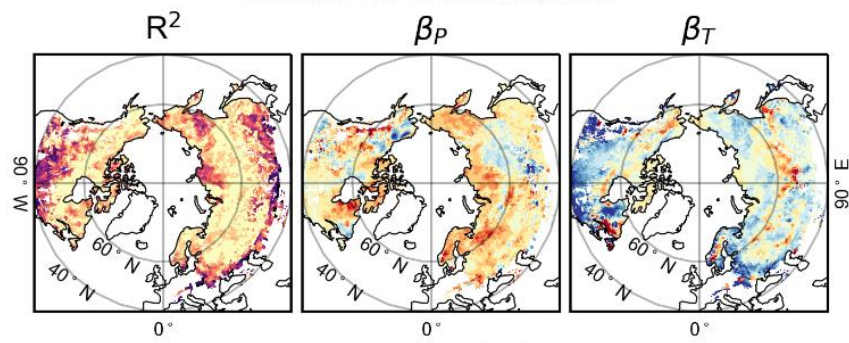

EC-Earth3-Veg historical

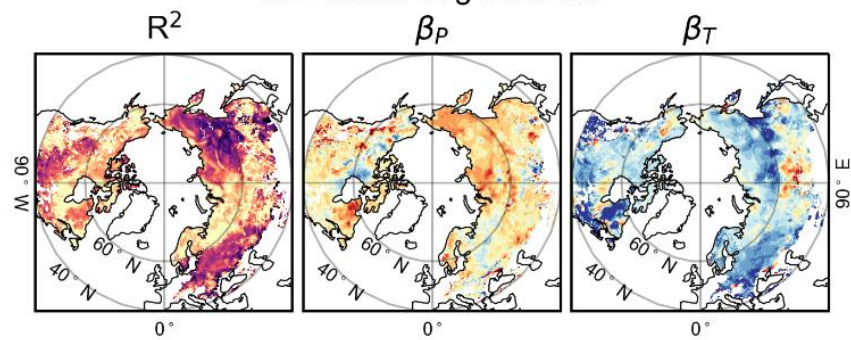

GFDL-ESM4 historical

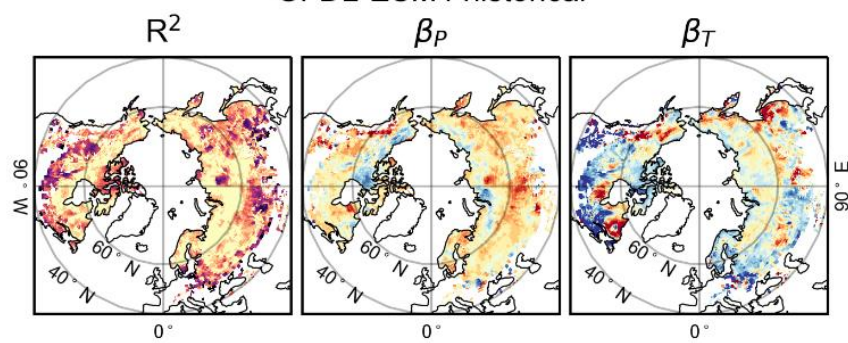

MPI-ESM1-2-HR historical

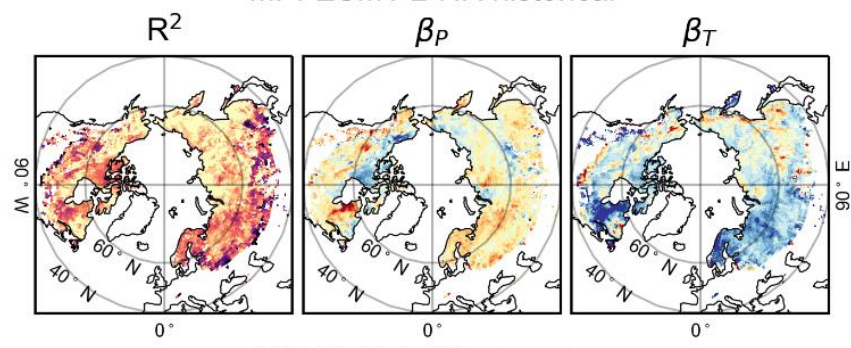

SAMO-UNICON historical
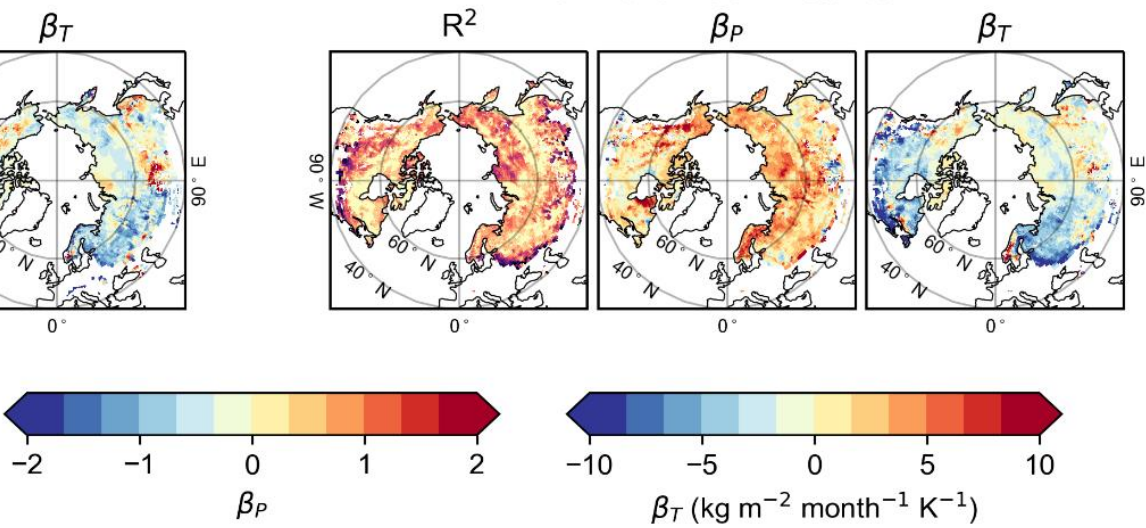

Figure S6. Linear regression parameters $\mathrm{R}^{2}, \beta_{\mathrm{P}}$, and $\beta_{\mathrm{T}}$ in spring 1982-2014. 
BCC-CSM2-MR historical

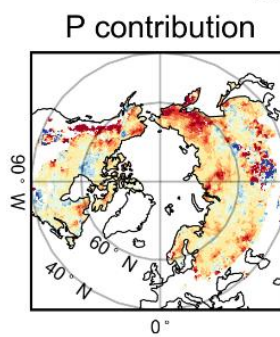

T contribution
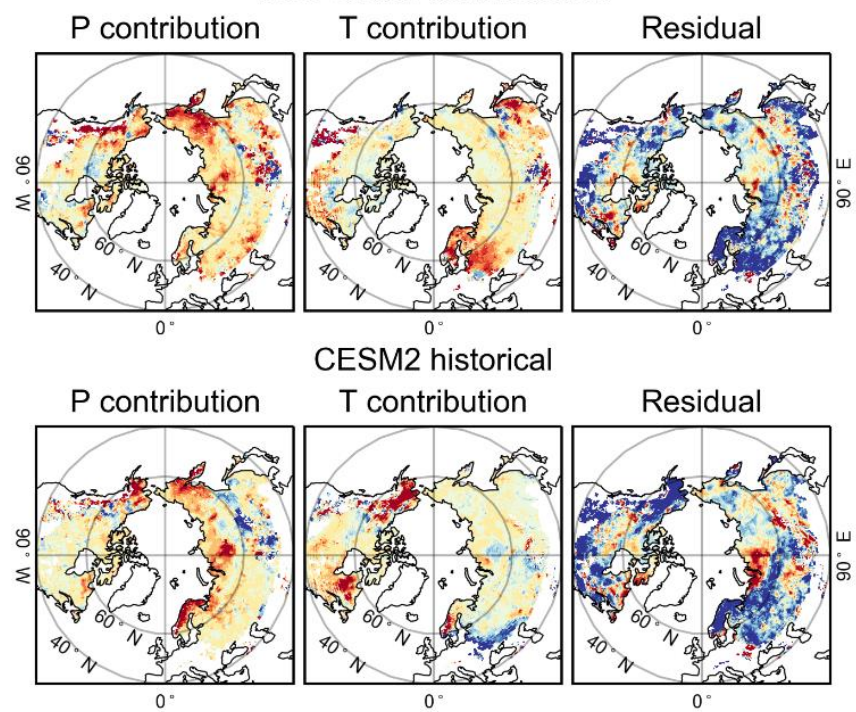

$\mathrm{T}$ contribution

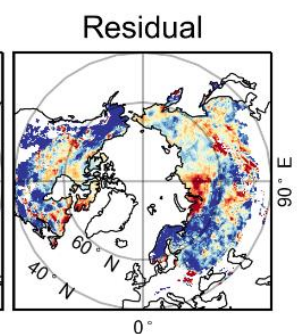

EC-Earth3 historical

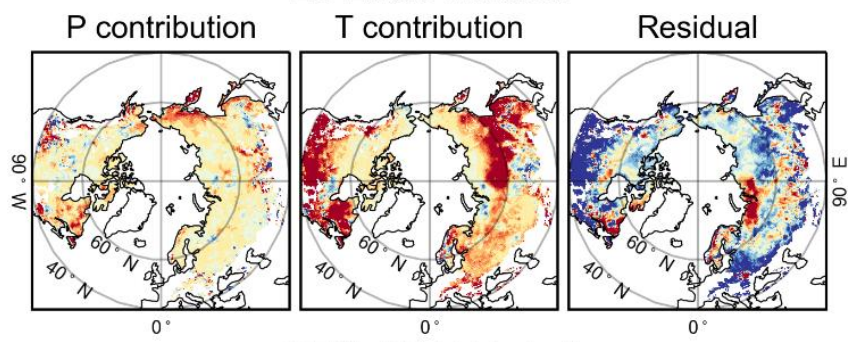

GFDL-CM4 historical

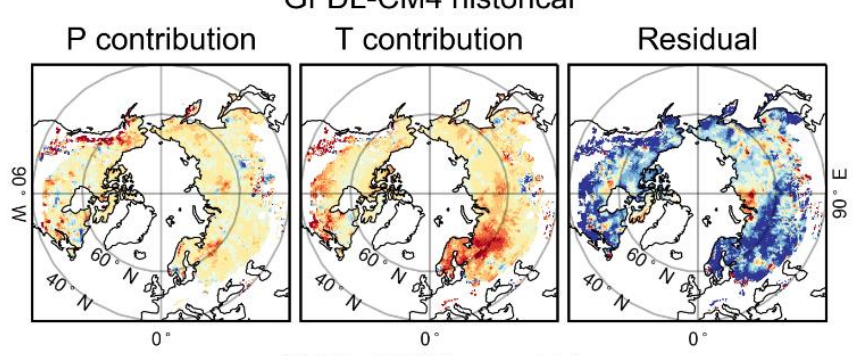

GFDL-ESM4 esm-hist

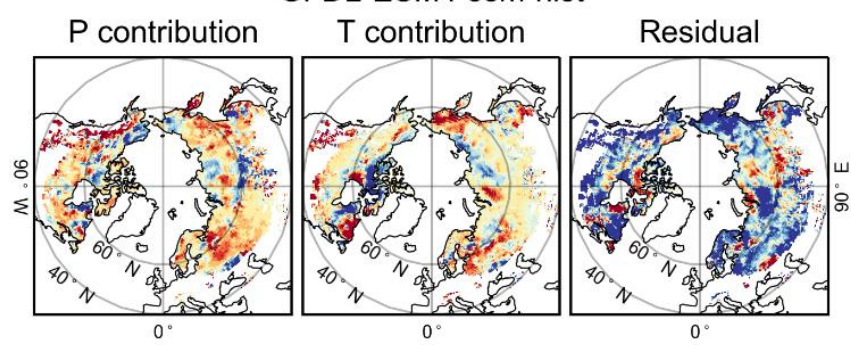

MRI-ESM2-0 historical
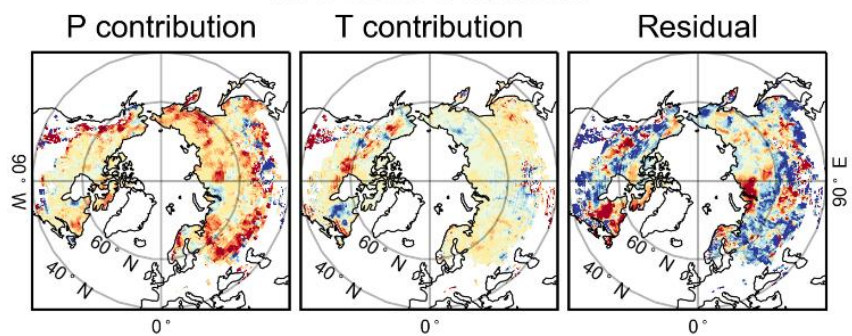

BCC-CSM2-MR esm-hist

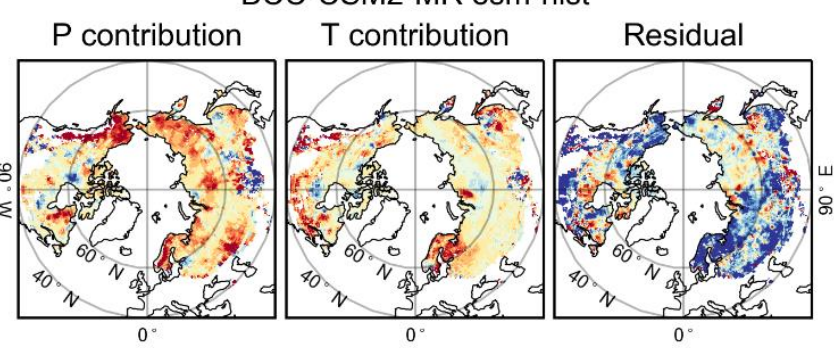

CESM2-WACCM historical

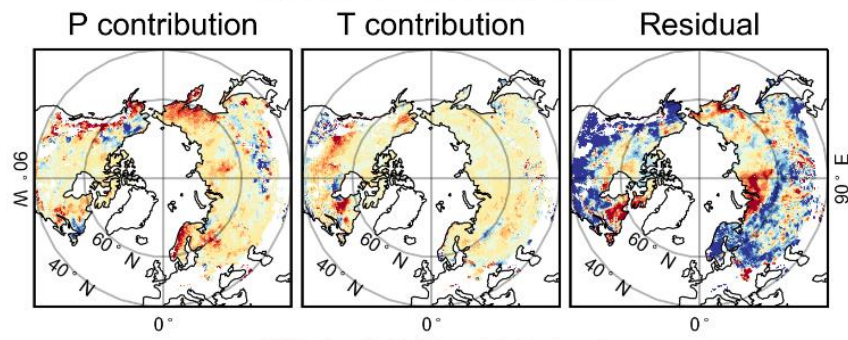

EC-Earth3-Veg historical

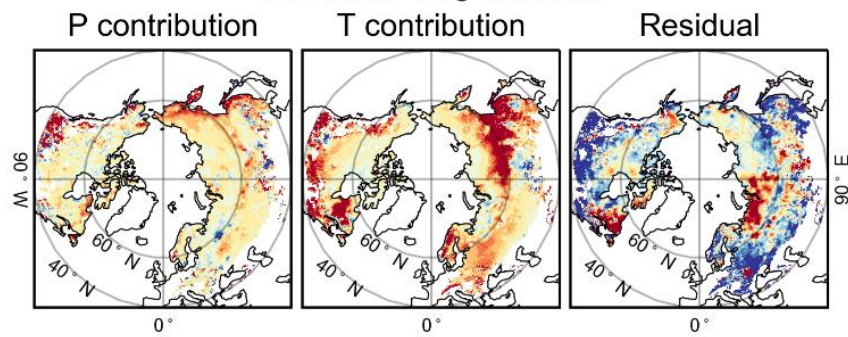

GFDL-ESM4 historical

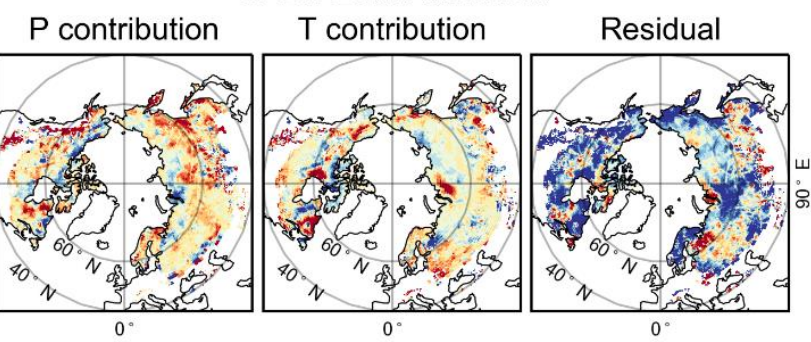

MPI-ESM1-2-HR historical
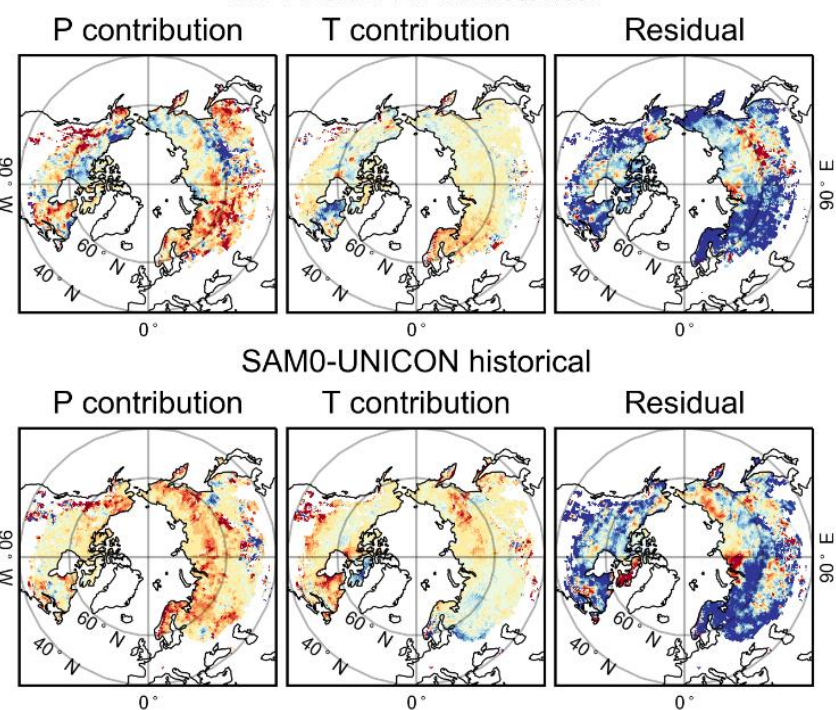

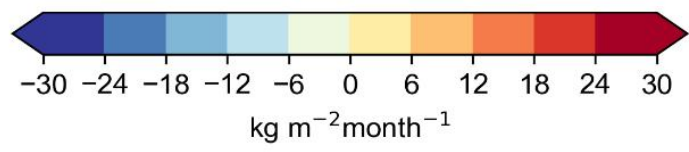

Figure S7. Spatial distribution of P contribution, T contribution and constant for each model in spring 1982-1991. 
BCC-CSM2-MR historical

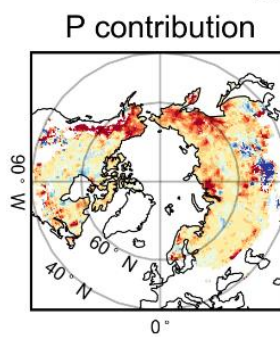

$T$ contribution
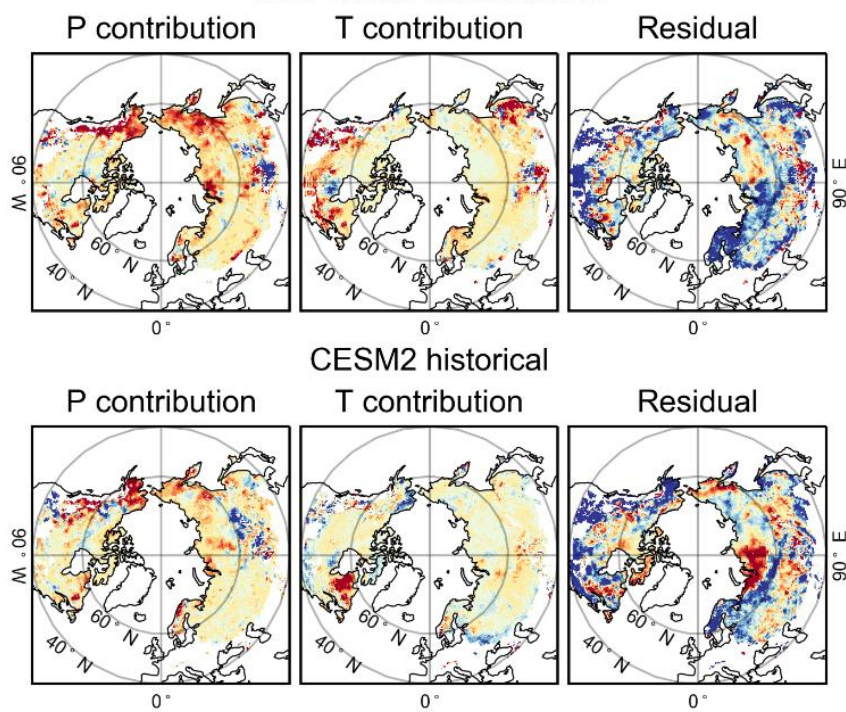

$\mathrm{T}$ contribution

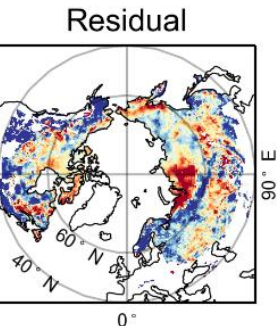

\section{EC-Earth3 historical}
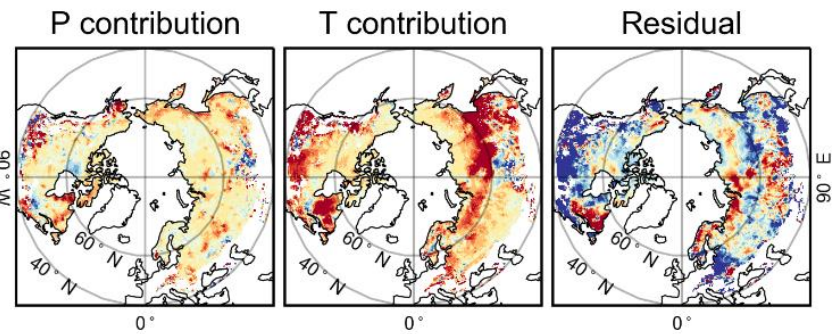

GFDL-CM4 historical

$P$ contribution $\mathrm{T}$ contribution
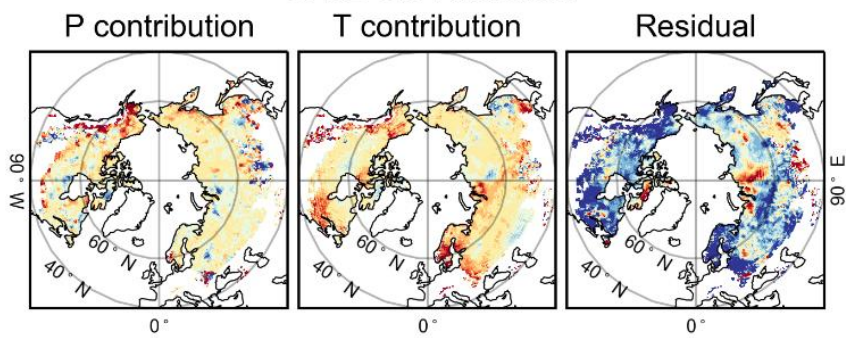

GFDL-ESM4 esm-hist

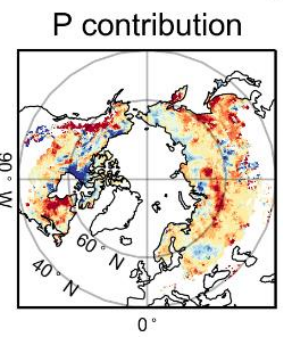

T contribution

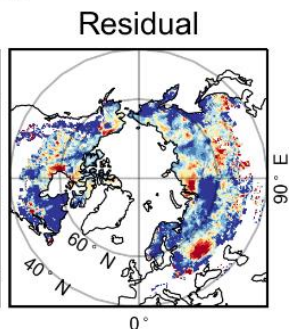

MRI-ESM2-0 historical
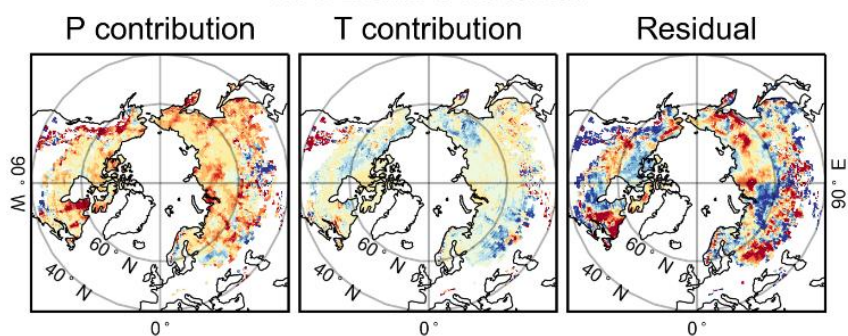

BCC-CSM2-MR esm-hist

$\mathrm{P}$ contribution $\mathrm{T}$ contribution Residual

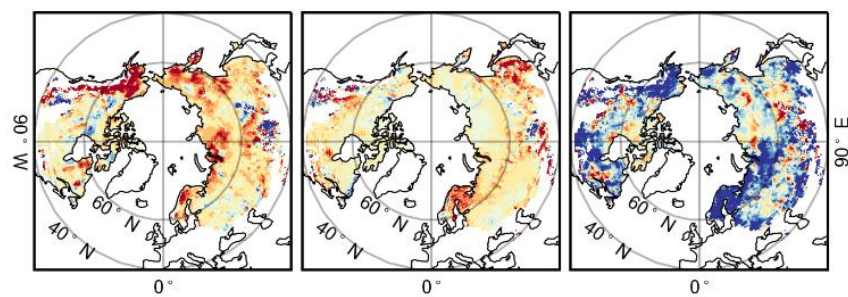

CESM2-WACCM historical

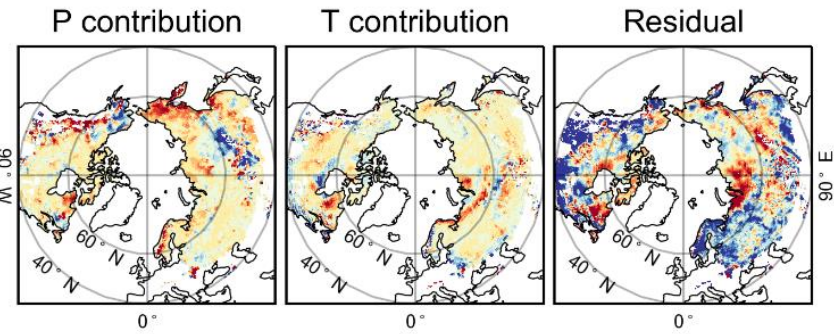

EC-Earth3-Veg historical

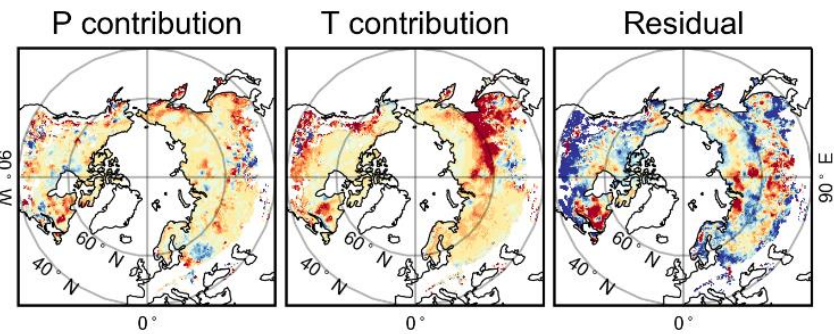

GFDL-ESM4 historical

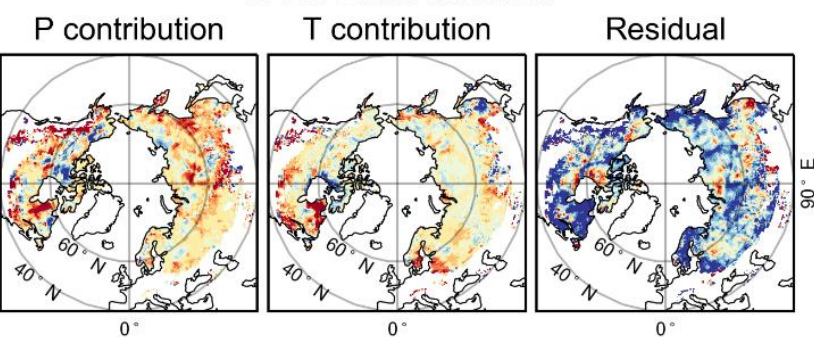

MPI-ESM1-2-HR historical
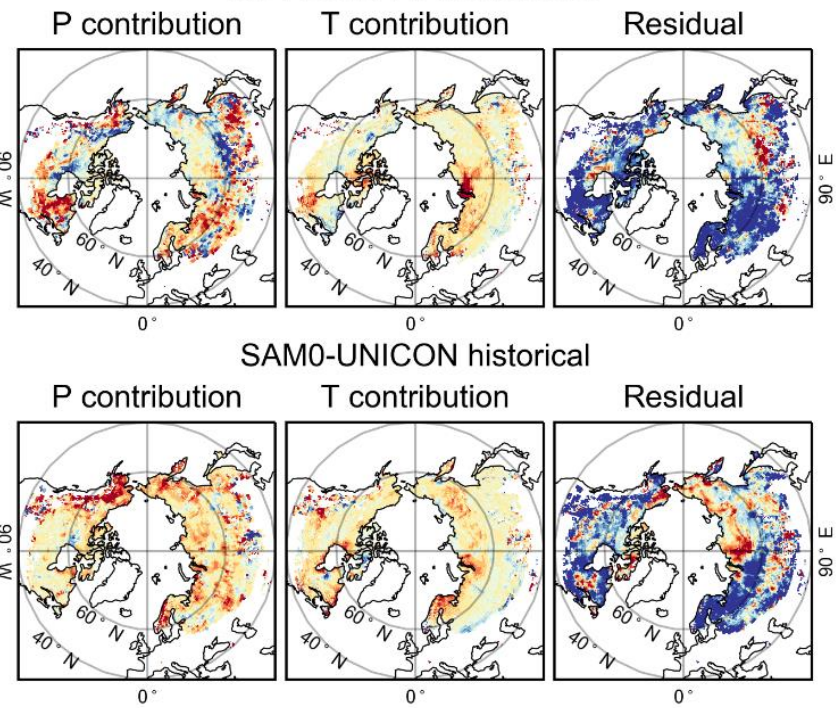

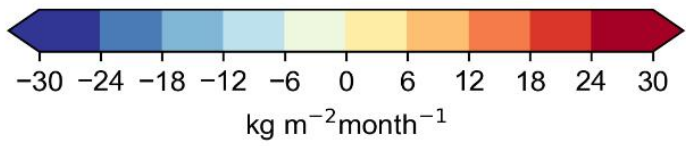

Figure S8. Spatial distribution of P contribution, T contribution and constant for each model in spring 1992-2001. 
BCC-CSM2-MR historical
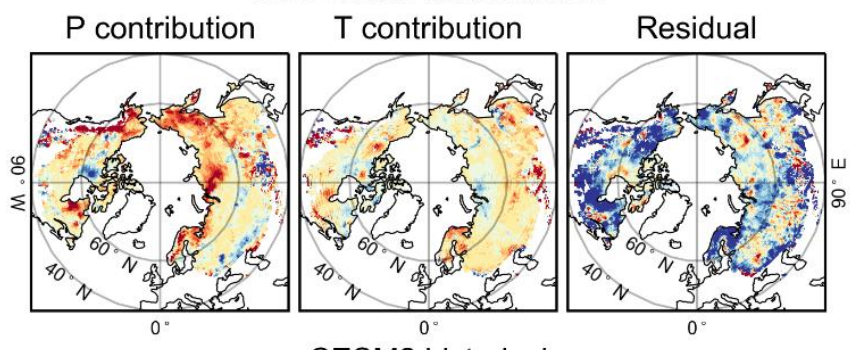

CESM2 historical

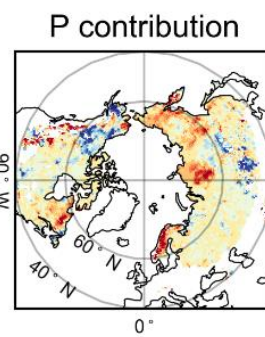

$T$ contribution

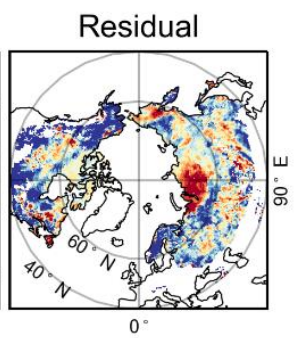

\section{EC-Earth3 historical}
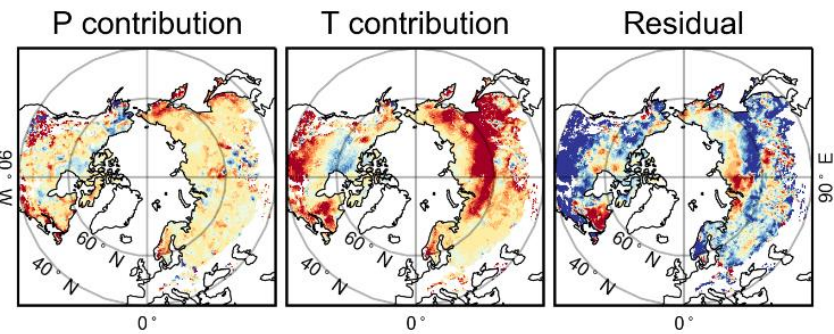

GFDL-CM4 historical

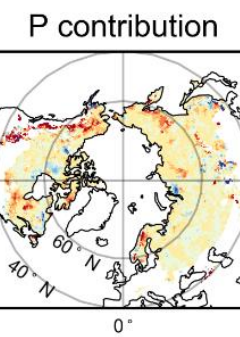

$\mathrm{T}$ contribution

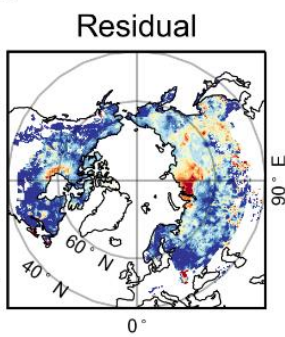

GFDL-ESM4 esm-hist

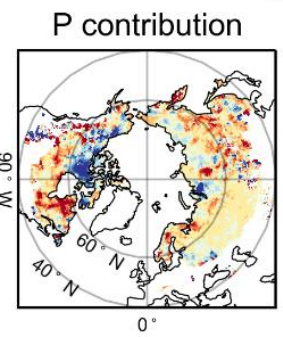

$T$ contribution

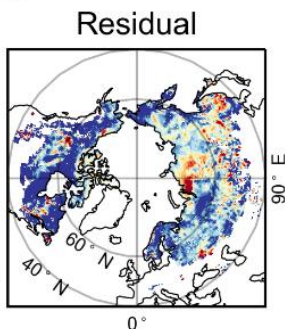

MRI-ESM2-0 historical
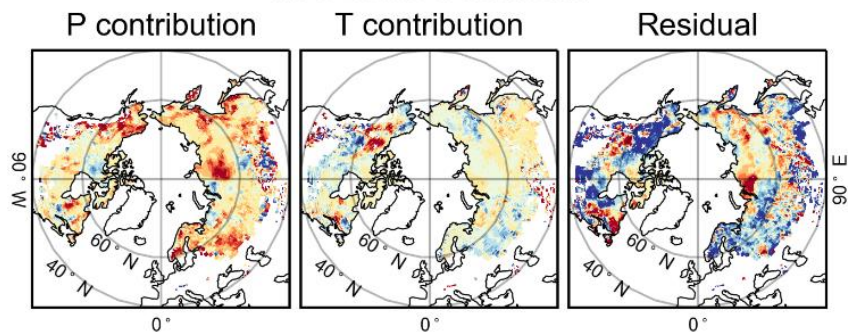

BCC-CSM2-MR esm-hist

$\mathrm{P}$ contribution $\quad \mathrm{T}$ contribution Residual

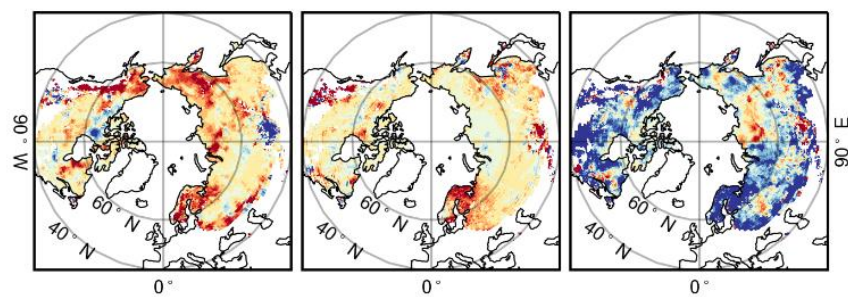

CESM2-WACCM historical

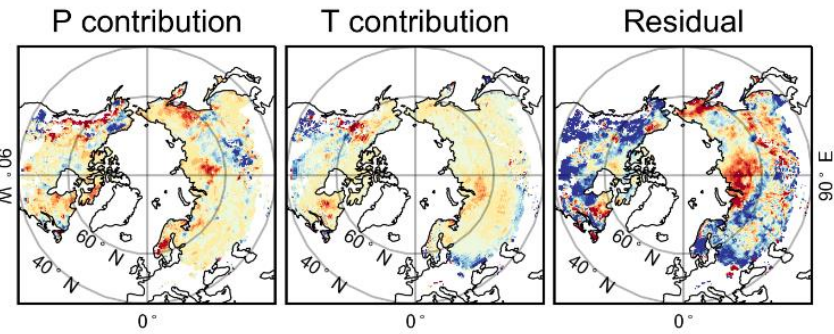

EC-Earth3-Veg historical

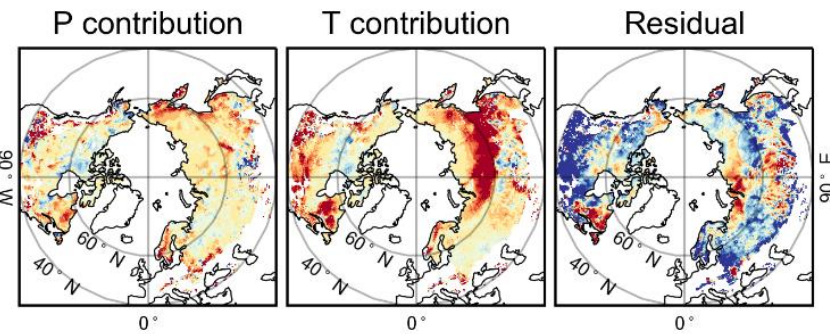

GFDL-ESM4 historical

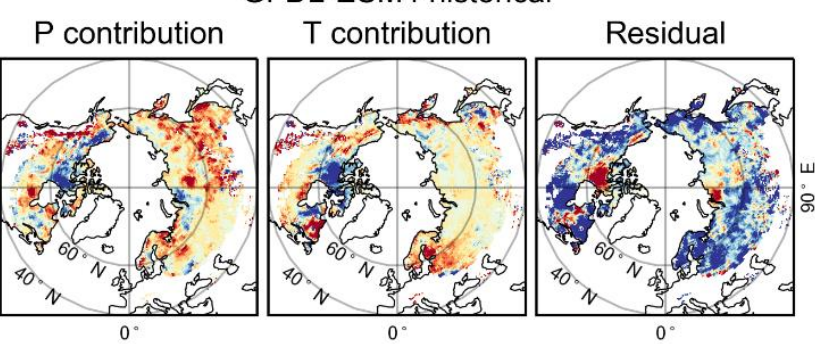

MPI-ESM1-2-HR historical
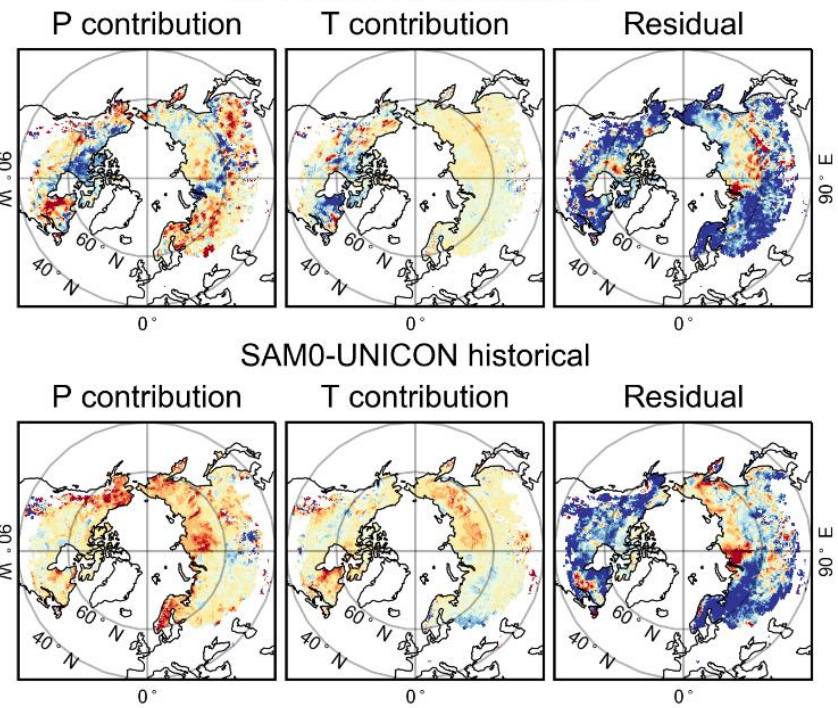

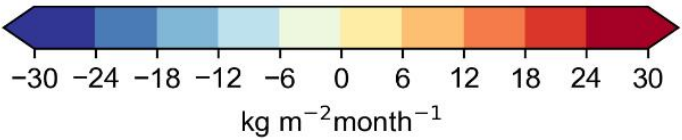

Figure S9. Spatial distribution of $\mathrm{P}$ contribution, T contribution and constant for each model in spring 2002-2014. 